\title{
Reflection and transmission coefficients of a single layer in poroelastic media
}

\author{
Robiel Martinez Corredor ${ }^{\mathrm{a})}$ \\ Facultad de Ingeniería, Universidad Nacional de La Plata, Calle 1 y 47, La Plata (B1900TAG), \\ Pcia. de Buenos Aires, Argentina \\ Juan E. Santos ${ }^{\text {b) }}$ \\ CONICET, Instituto del Gas y del Petróleo, Facultad de Ingeniería, Universidad de Buenos Aires, \\ Avenue Las Heras 2214 Piso 3, C1127AAR, Buenos Aires, Argentina \\ Patricia M. Gauzellino \\ Departamento de Geofísica Aplicada, Facultad de Ciencias Astronómicas y Geofísicas, Universidad Nacional \\ de La Plata, Paseo del Bosque s/n, La Plata, B1900FWA, Argentina \\ José M. Carcione \\ Istituto Nazionale di Oceanografia e di Geofisica Sperimentale (OGS), Borgo Grotta Gigante 42c, \\ 34010 Sgonico, Trieste, Italy
}

(Received 12 January 2014; revised 22 April 2014; accepted 28 April 2014)

\begin{abstract}
Wave propagation in poroelastic media is a subject that finds applications in many fields of research, from geophysics of the solid Earth to material science. In geophysics, seismic methods are based on the reflection and transmission of waves at interfaces or layers. It is a relevant canonical problem, which has not been solved in explicit form, i.e., the wave response of a single layer, involving three dissimilar media, where the properties of the media are described by Biot's theory. The displacement fields are recast in terms of potentials and the boundary conditions at the two interfaces impose continuity of the solid and fluid displacements, normal and shear stresses, and fluid pressure. The existence of critical angles is discussed. The results are verified by taking proper limits-zero and $100 \%$ porosity—by comparison to the canonical solutions corresponding to single-phase solid (elastic) media and fluid media, respectively, and the case where the layer thickness is zero, representing an interface separating two poroelastic half-spaces. As examples, it was calculated the reflection and transmission coefficients for plane wave incident at a highly permeable and compliant fluid-saturated porous layer, and the case where the media are saturated with the same fluid. (C) 2014 Acoustical Society of America. [http://dx.doi.org/10.1121/1.4875713]
\end{abstract}

PACS number(s): 43.20.Gp [OU]

Pages: 3151-3162

\section{INTRODUCTION}

The problem of reflection and refraction (transmission) of waves at a layer has practical applications in many fields. In geophysics, the topic is relevant in seismic methods, engineering and soil mechanics, hydrogeology, and underwater acoustics. In particular, in hydrocarbon exploration the layer is a porous medium, sandstone for instance, whose properties can be determined on the basis of multi-component seismic data, by using processing techniques, amplitude variations with offset (AVO) methods and inversion algorithms. The literature is vast in the case of a single interface. The authors attacked the problem for welded and non-welded interfaces (cracks and fractures), in some cases considering wave anisotropy and attenuation (Carcione, 1996, 1997, 1998;

\footnotetext{
a) Author to whom correspondence should be addressed. Electronic mail: robielmartinez@yahoo.com

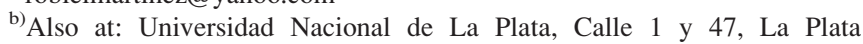
(B1900TAG), Pcia. de Buenos Aires, Argentina and Department of Mathematics, Purdue University, $150 \mathrm{~N}$. University Street, West Lafayette, IN 47907-2067.
}

Carcione and Picotti, 2012), and in the poroelastic case using Biot's theory (Santos et al., 1992), and a three-phase extension of this theory (Carcione et al., 2003; Rubino et al., 2006; Santos et al., 2004). There are relatively many works for a layer described by a single-phase (solid) case, e.g., Widess (1973) and Bakke and Ursin (1998) consider the normal incidence case for a thin layer, Juhlin and Young (1993) studied AVO effects of a thin layer, while the effect of the thickness of a sedimentary layer has been investigated by Chung and Lawton (1995a,b). Carcione (2001) computes the scattering response of a lossy layer having orthorhombic symmetry and embedded between two isotropic half-spaces, and Liu and Schmitt (2003) obtain the $P$-wave reflection coefficient in isotropic lossless media as a function of the incidence angle.

Ultrasonic properties of porous and permeable media have been treated by Wu et al. (1990), Johnson et al. (1994), Jocker and Smeulders (2009), and Fellah et al. (2013). In all these articles the authors have compared theoretical predictions with experimental data. In particular, Wu et al. (1990) studied the reflection and transmission of elastic waves at an interface between a fluid and a fluid-saturated porous 
medium and Fellah et al. (2013) analyzed fast and slow waves transmitted through human cancellous bone sample.

To our knowledge, the explicit calculation of the coefficients for poroelastic media has not been addressed. Existing methods are restricted to normal incidence and/or are based on numerical algorithms (Allard et al., 1986; Pride et al., 2002; Quintal et al., 2009; Schmidt and Tango, 1986). In general, these works are based on a constitutive equation described by Biot's theory of poroelasticity (Biot, 1956, 1962; Carcione, 2007; Carcione et al., 2010), which is sufficiently general to model the desired characteristics of wave propagation, in particular, the presence of the $P$ waves (typeI and type-II compressional waves) and its effects on interfaces (Dutta and Odé, 1979a,b, 1983; Plona, 1980).

We solve the scattering problem at all angles of incidence for a single layer embedded between two half-spaces with dissimilar media, where the properties of the media are described by Biot's theory of poroelasticity. The displacement fields are recast in terms of potentials and the boundary conditions at the two interfaces impose continuity of the solid and fluid displacements, normal and shear stresses, and fluid pressure. The methodology is analogous to that presented in Santos et al. (1992); Rubino et al. (2006); and Carcione (2001, 2007, Sec. 6.4). The results are verified for specific limiting cases with already published theoretical equations (Brekhovskikh, 1980; Carcione, 2007; Liu and Schmitt, 2003; Pilant, 1979; Santos et al., 1992).

The paper is organized as follows. Biot's theory is reviewed first. Then, we illustrate the methodology and finally we present the examples. The final equations are verified with limiting cases consisting of a single interface in poroelastic media and a layer, where the media can be solids or fluids. The examples are relevant for applications in reflection seismology.

\section{BIOT'S THEORY}

We consider a porous solid saturated by a viscous compressible fluid and assume that the whole aggregate is isotropic. Let $U$ and $U_{f}$ be the averaged displacement vectors of the solid and fluid parts of the medium, respectively. Then, $W$ is defined as the averaged relative fluid displacement per unit volume of bulk material,

$$
W=\phi\left(U_{f}-U\right),
$$

where $\phi$ is the effective porosity.

Let $\varepsilon_{i j}$ and $\sigma_{i j}$ denote the strain tensors of the solid and the bulk material, respectively, and let $P_{f}$ denote the fluid pressure. Following Biot $(1956,1962)$, the stress-strain relations can be written as

$$
\begin{aligned}
& \sigma_{\mathrm{ij}}=2 \mu \varepsilon_{\mathrm{ij}}(U)+\delta_{\mathrm{ij}}\left(\lambda_{c} \nabla \cdot U+D \nabla \cdot W\right), \quad i, j=1,2,3, \\
& P_{f}=-D \nabla \cdot U-M \nabla \cdot W
\end{aligned}
$$

(Carcione, 2007). Here, $\mu$ is the wet-rock shear modulus of the bulk material, considered to be equal to the shear modulus of the dry-rock. The grains are characterized by density $\rho_{s}$, bulk modulus $K_{s}$ and shear modulus $\mu_{s}$, while the fluid by $\rho_{f}, K_{f}$, and viscosity $\eta$. The grains are assumed to form an elastic porous matrix characterized by a porosity $\phi$, permeability $\kappa$, bulk modulus $K_{m}$, and shear modulus $\mu_{m}$. The Lamé constants of the saturated rock are $\lambda_{c}$ and $\mu$. The constants $\lambda_{c}, D$, and $M$ in Eq. (2) can be written as (Carcione, 2007; Dutta and Odé, 1979a,b, 1983)

$$
\begin{aligned}
& \alpha=1-\frac{K_{m}}{K_{s}}, \quad M=\left(\frac{\alpha-\phi}{K_{s}}+\frac{\phi}{K_{f}}\right)^{-1}, \quad D=\alpha M, \\
& K_{c}=K_{m}+\alpha^{2} M, \quad \lambda_{c}=K_{c}-\frac{2}{3} \mu .
\end{aligned}
$$

Next, let

$$
\rho_{b}=(1-\phi) \rho_{s}+\phi \rho_{f}
$$

be the mass density of the bulk material. Also, let $g$ and $b$ denote the mass and viscous coupling coefficients between the solid and fluid phases (Berryman, 1980, 1982; Dutta and Odé, 1979a,b, 1983):

$$
g=\frac{S \rho_{f}}{\phi}, \quad b=\frac{\eta}{\kappa}, \quad S=\frac{1}{2}\left(1+\frac{1}{\phi}\right),
$$

where $S$ is known as the structure factor. Then, assuming constant coefficients $\mu, \lambda_{c}, D$, and $M$ in Eq. (2), Biot's equations of motion can be stated as (Biot, 1956, 1962; Carcione, 2007)

$$
\begin{aligned}
\nabla \cdot \sigma & =H_{c} \nabla(\nabla \cdot U)-\mu \nabla \times(\nabla \times U)+D \nabla(\nabla \cdot W) \\
& =\rho_{b} \frac{\partial^{2} U}{\partial t^{2}}+\rho_{f} \frac{\partial^{2} W}{\partial t^{2}} \\
-\nabla P_{f} & =D \nabla(\nabla \cdot U)+M \nabla(\nabla \cdot W) \\
& =\rho_{f} \frac{\partial^{2} U}{\partial t^{2}}+g \frac{\partial^{2} W}{\partial t^{2}}+b \frac{\partial W}{\partial t}
\end{aligned}
$$

where $H_{c}=\lambda_{c}+2 \mu$.

A plane wave analysis shows that in this type of media two compressional waves (type-I and type-II waves) and one shear of $S$-wave can propagate (Biot, 1956).

\section{REFLECTION AND TRANSMISSION COEFFICIENTS}

The fluid-saturated system consists of three media, $\Omega_{n}$, $n=1,2,3$ with different properties as shown in Fig. 1 . Let $z=0$ be the boundary between $\Omega_{1}$ and $\Omega_{2}$, and $z=h$ the boundary between $\Omega_{2}$ and $\Omega_{3}$, and consider a type-I compressional plane wave in $\Omega_{1}$ incident at $z=0$ with an angle $\theta_{i 1}$ with respect to the vertical $z$ axis. Following Santos et al. (2004) and Dutta and Odé (1983), we represent the incident, reflected, and transmitted waves using potentials.

For $\Omega_{1}$ the potentials of the solid and relative fluid displacement are given by

$$
\begin{aligned}
& \varphi_{i 1}=A_{i 1} e^{\mathrm{i}\left(\omega t-\mathbf{q}_{i 1} \cdot \mathbf{x}\right)}, \\
& \psi_{i 1}=B_{i 1} e^{\mathrm{i}\left(\omega t-\mathbf{q}_{i 1} \cdot \mathbf{x}\right)},
\end{aligned}
$$

where 


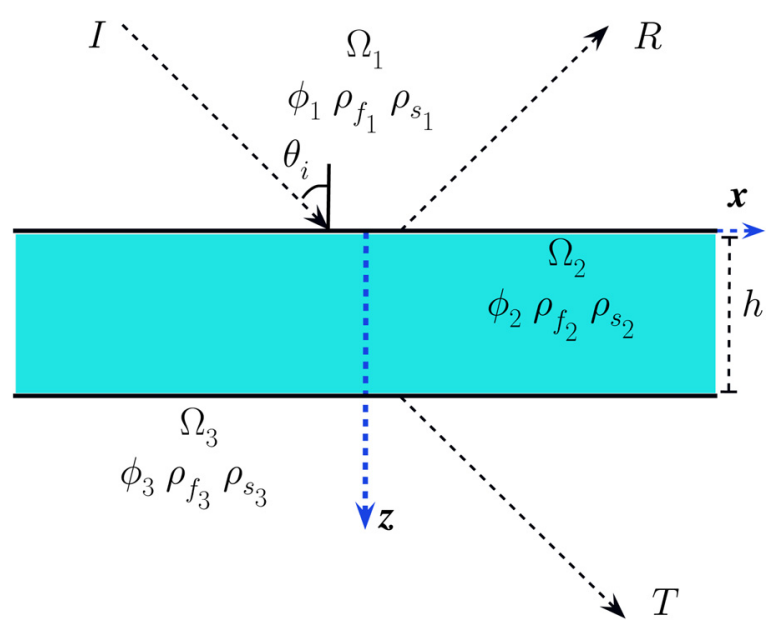

FIG. 1. (Color online) Geometry of the two half-spaces and the embedded layer.

$$
\mathbf{q}_{i 1}=q_{i 1}\left[\sin \left(\theta_{i 1}\right), \cos \left(\theta_{i 1}\right)\right]
$$

is the complex wave vector determining the polarization direction.

Let $\varphi_{r c}^{(1)}, \varphi_{r s}^{(1)}, \psi_{r c}^{(1)}$, and $\psi_{r s}^{(1)}$ be the compressional and shear potentials of the solid and relative fluid displacement, for the reflected waves in $\Omega_{1}$. They are given by

$$
\begin{aligned}
& \varphi_{r c}^{(1)}=A_{r 1}^{(1)} e^{\mathrm{i}\left(\omega t-\mathbf{q}_{r 1}^{(1)} \cdot \mathbf{x}\right)}+A_{r 2}^{(1)} e^{\mathrm{i}\left(\omega t-\mathbf{q}_{r 2}^{(1)} \cdot \mathbf{x}\right)}, \\
& \varphi_{r s}^{(1)}=A_{r s}^{(1)} e^{\mathrm{i}\left(\omega t-\mathbf{q}_{r s}^{(1)} \cdot \mathbf{x}\right)}, \\
& \psi_{r c}^{(1)}=B_{r 1}^{(1)} e^{\mathrm{i}\left(\omega t-\mathbf{q}_{r 1}^{(1)} \cdot \mathbf{x}\right)}+B_{r 2}^{(1)} e^{\mathrm{i}\left(\omega t-\mathbf{q}_{r 2}^{(1)} \cdot \mathbf{x}\right)}, \\
& \psi_{r s}^{(1)}=B_{r s}^{(1)} e^{\mathrm{i}\left(\omega t-\mathbf{q}_{r s}^{(1)} \cdot \mathbf{x}\right)},
\end{aligned}
$$

where the subscript $r$ indicates the reflected wave, $c$ indicates compressional wave and $s$ shear wave, the super-index (1) refers to medium 1 . Subscripts 1 and 2 indicate type-I and type-II waves, respectively.

In $\Omega_{2}$, the potentials are

$$
\begin{aligned}
& \varphi_{t c}^{(2)}=A_{t 1}^{(2)} e^{\mathrm{i}\left(\omega t-\mathbf{q}_{t 1}^{(2)} \cdot \mathbf{x}\right)}+A_{t 2}^{(2)} e^{\mathrm{i}\left(\omega t-\mathbf{q}_{t 2}^{(2)} \cdot \mathbf{x}\right)}, \\
& \varphi_{t s}^{(2)}=A_{t s}^{(2)} e^{\mathrm{i}\left(\omega t-\mathbf{q}_{t s}^{(2)} \cdot \mathbf{x}\right)}, \\
& \psi_{t c}^{(2)}=B_{t 1}^{(2)} e^{\mathrm{i}\left(\omega t-\mathbf{q}_{t 1}^{(2)} \cdot \mathbf{x}\right)}+B_{t 2}^{(2)} e^{\mathrm{i}\left(\omega t-\mathbf{q}_{t 2}^{(2)} \cdot \mathbf{x}\right)}, \\
& \psi_{t s}^{(2)}=B_{t s}^{(2)} e^{\mathrm{i}\left(\omega t-\mathbf{q}_{t s}^{(2)} \cdot \mathbf{x}\right)}, \\
& \varphi_{r c}^{(2)}=A_{r 1}^{(2)} e^{\mathrm{i}\left(\omega t-\mathbf{q}_{r 1}^{(2)} \cdot \mathbf{x}\right)}+A_{r 2}^{(2)} e^{\mathrm{i}\left(\omega t-\mathbf{q}_{r 2}^{(2)} \cdot \mathbf{x}\right)}, \\
& \varphi_{r s}^{(2)}=A_{r s}^{(2)} e^{\mathrm{i}\left(\omega t-\mathbf{q}_{r s}^{(2)} \cdot \mathbf{x}\right)}, \\
& \psi_{r c}^{(2)}=B_{r 1}^{(2)} e^{\mathrm{i}\left(\omega t-\mathbf{q}_{r 1}^{(2)} \cdot \mathbf{x}\right)}+B_{r 2}^{(2)} e^{\mathrm{i}\left(\omega t-\mathbf{q}_{r 2}^{(2)} \cdot \mathbf{x}\right)}, \\
& \psi_{r s}^{(2)}=B_{r s}^{(2)} e^{\mathrm{i}\left(\omega t-\mathbf{q}_{r s}^{(2)} \cdot \mathbf{x}\right)},
\end{aligned}
$$

the subscript $t$ indicating the transmitted wave.

Finally, potentials in $\Omega_{3}$ are expressed by

$$
\begin{aligned}
& \varphi_{t c}^{(3)}=A_{t 1}^{(3)} e^{\mathrm{i}\left(\omega t-\mathbf{q}_{t 1}^{(3)} \cdot \mathbf{x}\right)}+A_{t 2}^{(3)} e^{\mathrm{i}\left(\omega t-\mathbf{q}_{t 2}^{(3)} \cdot \mathbf{x}\right)}, \\
& \varphi_{t s}^{(3)}=A_{t s}^{(3)} e^{\mathrm{i}\left(\omega t-\mathbf{q}_{t s}^{(3)} \cdot \mathbf{x}\right)}, \\
& \psi_{t c}^{(3)}=B_{t 1}^{(3)} e^{\mathrm{i}\left(\omega t-\mathbf{q}_{t 1}^{(3)} \cdot \mathbf{x}\right)}+B_{t 2}^{(3)} e^{\mathrm{i}\left(\omega t-\mathbf{q}_{t 2}^{(3)} \cdot \mathbf{x}\right)}, \\
& \psi_{t s}^{(3)}=B_{t s}^{(3)} e^{\mathrm{i}\left(\omega t-\mathbf{q}_{t s}^{(3)} \cdot \mathbf{x}\right)} .
\end{aligned}
$$

In general, we determine $\mathbf{q}_{l j}=\left(\chi_{l j}, \beta_{l j}\right)=q_{l j}\left[\sin \left(\theta_{l j}\right)\right.$, $\left.\cos \left(\theta_{l j}\right)\right], l=i, r, t$, and $j=1,2, s$ for each kind of wave.

The solid and relative fluid vectors $U^{(n)}=\left(U_{x}^{(n)}, U_{z}^{(n)}\right)$ and $W^{(n)}=\left(W_{x}^{(n)}, W_{z}^{(n)}\right)$ in $\Omega_{n}, n=1,2,3$ are given by (Santos et al., 1992)

$$
\begin{aligned}
U^{(1)}= & \nabla \varphi_{i 1}+\nabla \varphi_{r c}^{(1)}+\left(-\frac{\partial \varphi_{r s}^{(1)}}{\partial z}, \frac{\partial \varphi_{r s}^{(1)}}{\partial x}\right) \\
= & U_{i 1}^{(1)}+U_{r 1}^{(1)}+U_{r 2}^{(1)}+U_{r s}^{(1)}, \\
W^{(1)}= & \nabla \psi_{i 1}+\nabla \psi_{r c}^{(1)}+\left(-\frac{\partial \psi_{r s}^{(1)}}{\partial z}, \frac{\partial \psi_{r s}^{(1)}}{\partial x}\right) \\
= & W_{i 1}^{(1)}+W_{r 1}^{(1)}+W_{r 2}^{(1)}+W_{r s}^{(1)}, \\
U^{(2)}= & \nabla \varphi_{t c}^{(2)}+\left(-\frac{\partial \varphi_{t s}^{(2)}}{\partial z}, \frac{\partial \varphi_{t s}^{(2)}}{\partial x}\right)+\nabla \varphi_{r c}^{(2)} \\
& +\left(-\frac{\partial \varphi_{r s}^{(2)}}{\partial z}, \frac{\partial \varphi_{r s}^{(2)}}{\partial x}\right) \\
= & U_{t 1}^{(2)}+U_{t 2}^{(2)}+U_{t s}^{(2)}+U_{r 1}^{(2)}+U_{r 2}^{(2)}+U_{r s}^{(2)}, \\
W^{(3)}= & \nabla \psi_{r c}^{(3)}+\left(-\frac{\partial \psi_{r s}^{(3)}}{\partial z}, \frac{\partial \psi_{r s}^{(3)}}{\partial x}\right) \\
= & W_{t 1}^{(3)}+W_{t 2}^{(3)}+W_{t s}^{(3)} \cdot \\
W^{(2)}= & \nabla \psi_{t c}^{(1)}+\left(-\frac{\partial \psi_{t s}^{(2)}}{\partial z}, \frac{\partial \psi_{t s}^{(2)}}{\partial x}\right)+\nabla \psi_{r c}^{(2)} \\
& +\left(-\frac{\partial \psi_{r s}^{(2)}}{\partial z}, \frac{\partial \psi_{r s}^{(2)}}{\partial x}\right) \\
= & \left.W_{t 1}^{(2)}+W_{t 2}^{(2)}+W_{t s}^{(2)}+W_{r 1}^{(3)}+U_{t 2}^{(3)}+U_{t s}^{(3)}, \frac{\partial \varphi_{t s}^{(3)}}{\partial z}\right) \\
(3 x & W_{r s}^{(2)},
\end{aligned}
$$

Here $U_{l j}^{(n)}$ and $W_{l j}^{(n)}, l=i, r, t, j=1,2, s$, denote the type-I $P$ wave, type-II $P$ wave, and shear wave components of $U^{(n)}$ and $W^{(n)}$, respectively. The super-index $(n)$ denotes any variable associated with the medium $\Omega_{n}$.

The boundary conditions at the interfaces located at $z=0$ and $z=h$ impose continuity of the solid and fluid displacements, continuity of the normal and shear stress, and continuity of the fluid pressure (Dutta and Odé, 1983; Santos et al., 2004). Therefore, at $z=0$ and $z=h$ we impose the conditions

$$
\begin{aligned}
& U_{x}^{(n)}=U_{x}^{(n+1),} \\
& U_{z}^{(n)}=U_{z}^{(n+1),} \\
& \sigma_{z z}^{(n)}=\sigma_{z z}^{(n+1)}, \\
& \sigma_{x z}^{(n)}=\sigma_{x z}^{(n+1)}, \\
& P_{f}^{(n)}=P_{f}^{(n+1)},
\end{aligned}
$$




$$
W_{z}^{(n)}=W_{z}^{(n+1)}, \quad n=1,2 .
$$

The amplitude of the reflection and transmission coefficients $R_{j}^{(1)}$ and $T_{j}^{(3)}, j=1,2, s$, for the different waves are defined as the ratio of the solid-displacement amplitude of the corresponding wave and that of the incident wave (Dutta and Odé, 1983; Santos et al., 2004), i.e,

$$
R_{j}^{(1)}=\frac{A_{r j}^{(1)} q_{r j}^{(1)}}{A_{i 1}^{(1)} q_{i 1}^{(1)}}
$$

and

$$
T_{j}^{(3)}=\frac{A_{t j}^{(3)} q_{t j}^{(3)}}{A_{i 1}^{(1)} q_{i 1}^{(1)}} .
$$

Using Eqs. (7)-(10) to obtain expressions for each of the pairs mentioned above and substituting them in Eq. (6) leads us to the following relationships between the amplitudes of the solid and the relative amplitudes to the fluid (Santos et al., 2004):

$$
\begin{aligned}
& B_{l j}^{(n)}=\gamma_{l j}^{(n)} A_{l j}^{(n)}, \quad j=1,2, s, \quad l=r, t, \quad n=1,2,3, \\
& B_{i 1}=\gamma_{i 1} A_{i 1}
\end{aligned}
$$

with

$$
\begin{aligned}
& \gamma_{r j}^{(n)}=\frac{\left[\rho_{b}^{(n)} \omega^{2}-\left(q_{r j}^{(n)}\right)^{2} H_{c}^{(n)}\right]}{\left[\left(q_{r j}^{(n)}\right)^{2} D^{(n)}-\rho_{f}^{(n)} \omega^{2}\right]}, j=1,2, \quad n=1,2, \\
& \gamma_{i 1}^{(n)}=\frac{\left[\rho_{b}^{(1)} \omega^{2}-\left(q_{i 1}^{(1)}\right)^{2} H_{c}^{(1)}\right]}{\left[\left(q_{i 1}^{(1)}\right)^{2} D^{(1)}-\rho_{f}^{(1)} \omega^{2}\right]}, \\
& \gamma_{t j}^{(n)}=\frac{\left[\rho_{b}^{(n)} \omega^{2}-\left(q_{t j}^{(n)}\right)^{2} H_{c}^{(n)}\right]}{\left[\left(q_{t j}^{(n)}\right)^{2} D^{(n)}-\rho_{f}^{(n)} \omega^{2}\right]}, j=1,2, \quad n=2,3, \\
& \gamma_{r s}^{(n)}=\frac{\mu^{(n)}\left(q_{r s}^{(n)}\right)^{2}-\rho_{b}^{(n)} \omega^{2}}{\rho_{f}^{(n)} \omega^{2}}, n=1,2, \\
& \gamma_{t s}^{(n)}=\frac{\mu^{(n)}\left(q_{t s}^{(n)}\right)^{2}-\rho_{b}^{(n)} \omega^{2}}{\rho_{f}^{(n)} \omega^{2}}, n=2,3 .
\end{aligned}
$$

The boundary conditions (17)-(22) require that the phase factors at the interfaces $z=0$ and $z=h$ are the same:

$$
\begin{aligned}
\chi_{i 1} & =\chi_{r 1}^{(1)}=\chi_{r 2}^{(1)}=\chi_{r s}^{(1)}=\chi_{t 1}^{(2)}=\chi_{t 2}^{(2)}=\chi_{t s}^{(2)}=\chi_{r 1}^{(2)} \\
& =\chi_{r 2}^{(2)}=\chi_{r s}^{(2)}=\chi_{t 1}^{(3)}=\chi_{t 2}^{(3)}=\chi_{t s}^{(3)}=\chi,
\end{aligned}
$$

which represents Snell's law and allows us to obtain the reflected and transmitted angles $\theta_{l j}$ for each type of wave as a function of the incidence angle $\theta_{i 1}$.
Application of the boundary conditions (17)-(22) and Snell's law Eq. (26) at $z=0$ and $z=h$ give two systems of linear equations in the unknowns $A_{r 1}, A_{r 2}, A_{r s}, A_{t 1}, A_{t 2}$, and $A_{t s}$ (see Appendix A). These two systems have coefficients depending on the wave numbers $q_{l j}^{(n)}, \quad n=1,2,3$, $l=i, r, t, j=1,2, s$.

Set

$$
C_{l j}^{(n)}=A_{l j}^{(n)} / A_{i 1}, \quad l=r, t, \quad j=1,2, s, \quad n=1,2,3 .
$$

Using the matrix notation of Carcione (2007, Sec. 6.4) to relate the fields at $z=0$ and $z=h$ we obtain

$$
\left(\mathbf{A}_{1}-\mathbf{B} * \mathbf{A}_{3}\right) \mathbf{r}=-\mathbf{i}_{p}
$$

where $\mathbf{r}=\left(C_{r 1}^{(1)}, C_{r 2}^{(1)}, C_{r s}^{(1)}, C_{t 1}^{(3)}, C_{t 2}^{(3)}, C_{t s}^{(3)}\right)^{\mathrm{T}}, \mathbf{i}_{p}=\left[-\chi,-\beta_{i 1}^{(1)}\right.$, $\left.\zeta_{i 1}^{(1)},-2 \mu^{(1)} \chi \beta_{i 1}^{(1)}, \xi_{i 1}^{(1)},-\beta_{i 1}^{(1)} \gamma_{i 1}^{(1)}\right]^{\mathrm{T}}$, and $\mathbf{B}=\mathbf{T}(0) *[\mathbf{T}(h)]^{-1}$ that acts as a boundary condition. The matrices of the system Eq. (28) are given in Appendix B.

The amplitude of the reflection and transmission coefficients for the different types of waves are defined as

$$
\begin{aligned}
& R_{j}^{(1)}=C_{r j}^{(1)} \frac{q_{r j}^{(1)}}{q_{i 1}^{(1)}}, \\
& T_{j}^{(3)}=C_{t j}^{(3)} \frac{q_{t j}^{(3)}}{q_{i 1}^{(1)}}, \quad j=1,2, s .
\end{aligned}
$$

An incident $S$ wave has the same scattering matrix as the $P$ incident wave, but the array $\mathbf{i}_{p}$ in Eq. (28) is replaced by

$$
\mathbf{i}_{s}=\left[\beta_{i s}^{(1)},-\chi,-\zeta_{i s}^{(1)},-\mu^{(1)}\left\{\chi^{2}-\left(\beta_{i s}^{(1)}\right)^{2}\right\}, 0,-\gamma_{i s}^{(1)} \chi\right]^{\mathrm{T}} .
$$

\section{EXAMPLES}

In this section, we test the reflection and transmission coefficients and consider several cases of interest in reservoir geophysics. The following cases are taken into account.

Case 1: A validation test when $\Omega_{1}, \Omega_{2}$, and $\Omega_{3}$ are inviscid fluids, corresponding to the limit case $\phi=1, \mu=0, \eta=0$.

Case 2: A validation test when we have a single interface between two elastic isotropic solids $\Omega_{1}$ and $\Omega_{3}$, corresponding to the limit case $\phi=0, \eta=0$.

Case 3: A single porous medium saturated with three different fluids, so that we have three different media identified with $\Omega_{1}, \Omega_{2}$, and $\Omega_{3}$ as in Fig. 1 . The objective is to take the limit when the layer thickness $h$ of $\Omega_{2}$ tends to zero to recover the results obtained by Santos et al. (2004).

Case 4: A porous background with an embedded porous layer $\Omega_{2}$. The media are only saturated with water. The reflection and transmission coefficients are shown for three different values of the thickness of layer $h$.

\section{A. Case 1. $P$ waves in fluid media}

We check the reflection and transmission coefficients for compressional plane waves propagating within a fluid 
TABLE I. Properties of the saturant fluids.

\begin{tabular}{llll}
\hline \hline Property & Gas & Water & Oil \\
\hline$\rho_{f}\left(\mathrm{~g} / \mathrm{cm}^{3}\right)$ & 0.1398 & 1.0 & 0.7 \\
$K_{f}(\mathrm{GPa})$ & 0.05543 & 2.25 & 0.57 \\
$\eta(\mathrm{Poise})$ & 0.00022 & 0.01 & 0.04 \\
\hline
\end{tabular}

medium. Considering the notation in Fig. 1, we choose $\Omega_{1}$ as water, $\Omega_{2}$ as oil and $\Omega_{3}$ as gas, all with zero viscosity and with densities and bulk moduli given in Table I. To model $\Omega_{1}, \Omega_{2}$, and $\Omega_{3}$ as fluids, the shear modulus is set to zero, while the porosity is set to one. Thus, in this case only the boundary conditions (21) and (22) are considered. The thickness of the layer $\Omega_{2}$ is $h=0.1 \mathrm{~cm}$, which is equal to $0.0055 \%$ of the wavelength of the compressional wave in $\Omega_{2}$.

Figure 2 shows the magnitude and phase of the reflection and transmission coefficients versus the incidence
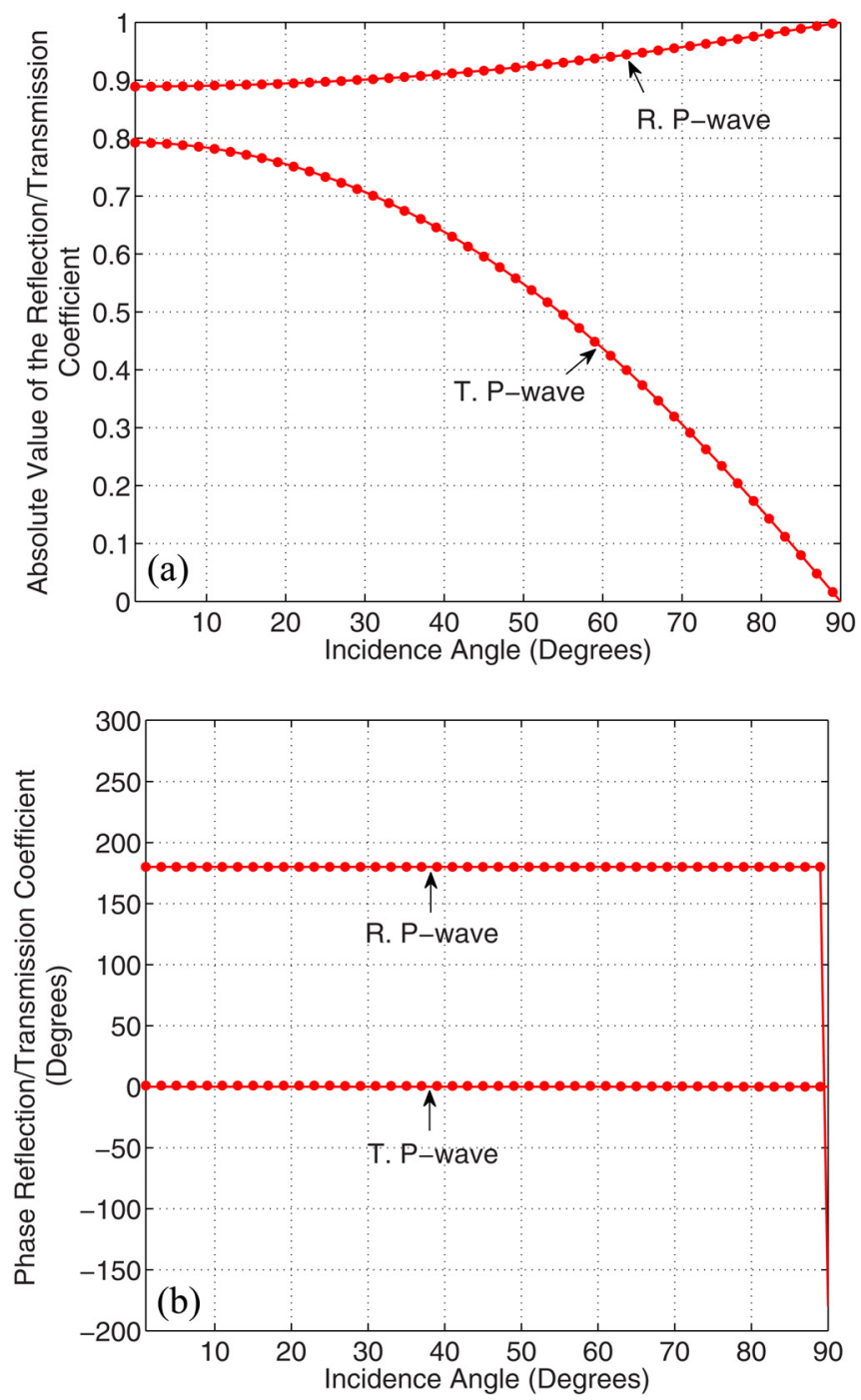

FIG. 2. (Color online) Reflection and transmission coefficients of the compressional wave as a function of the incidence angle for a thickness $h=0.1 \mathrm{~cm}$. The solid lines and dots correspond to Liu and Schmitt's solution and the present solution, respectively. The incident $P$ wave has a frequency of $50 \mathrm{~Hz}, \phi=1$ and $\eta=\mu=0$. (a) Absolute value of the reflection and transmission coefficients; (b) phase angle of the reflection and transmission coefficients. propagation angle. It can be observed a perfect agreement with the analytical results [Eqs. (A11) and (A12)] of Liu and Schmitt (2003).

\section{B. Case 2. $P$ and $S$ waves in solid media}

We choose $\Omega_{1}$ and $\Omega_{3}$ to be solids with $K_{s}=37 \mathrm{GPa}$, $\mu_{s}=44 \mathrm{GPa}$, and $\rho_{s}=2.65 \mathrm{~g} / \mathrm{cm}^{3}$, while the layer $\Omega_{2}$ is defined by $\rho_{s}=2.55 \mathrm{~g} / \mathrm{cm}^{3}, K_{s}=25 \mathrm{GPa}$, and $\mu_{s}=9 \mathrm{GPa}$. The porosity is set to zero, and therefore there are no pore fluids. The thickness of the layer is $h=0.1 \mathrm{~cm}$, corresponding to $0.0013 \%$ of the wavelength of the compressional wave in $\Omega_{2}$.

Figure 3 shows the reflection and transmission coefficients of compressional and shear waves due to the tetrapartition of the incident $P$-wave at the interfaces $z=0$ and
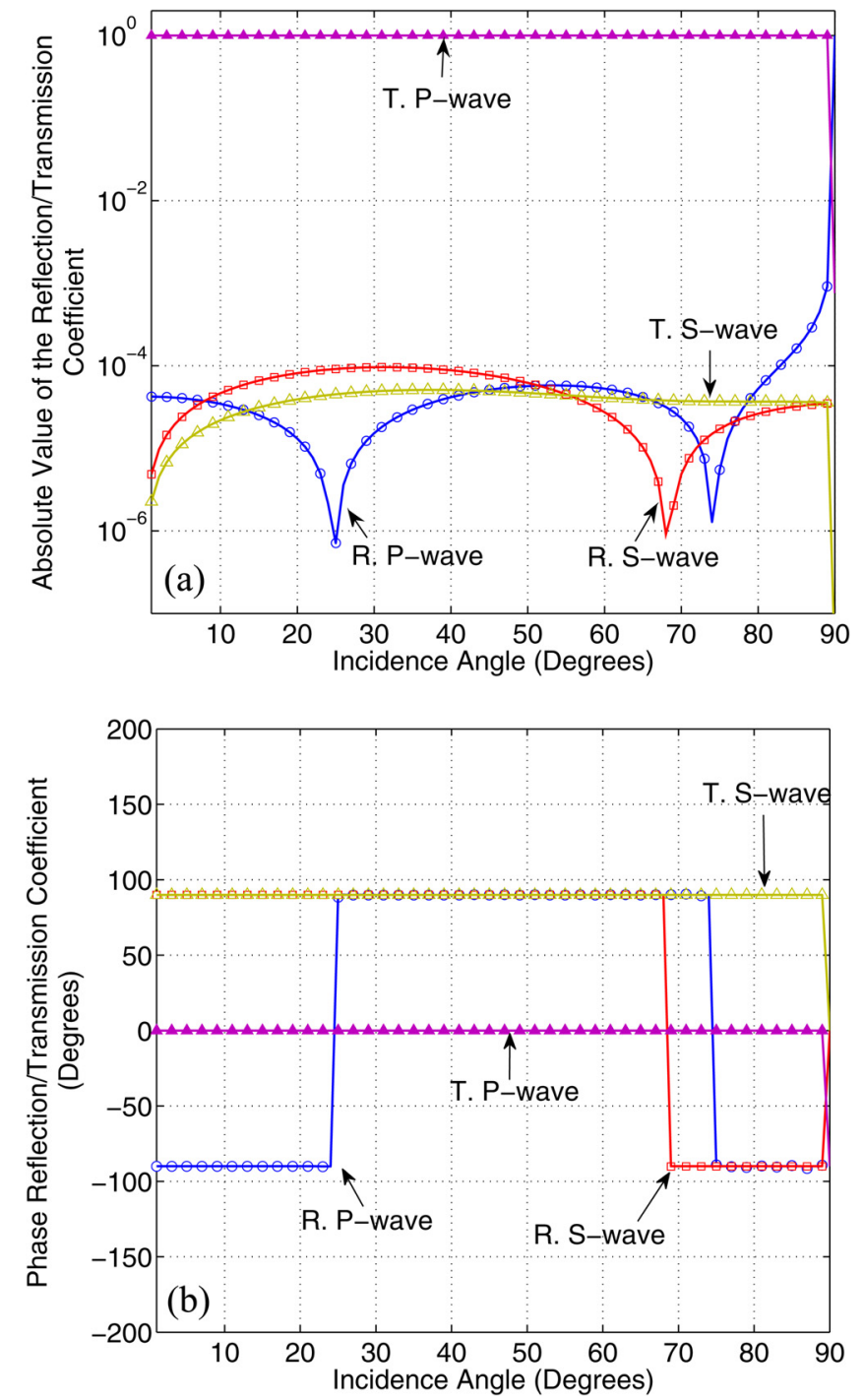

FIG. 3. (Color online) Reflection and transmission coefficients of the compressional and shear waves as a function of the incidence angle for a thickness $h=0.1 \mathrm{~cm}$. The solid and dotted lines correspond to Carcione's solution and the present solution, respectively. The incident $P$ wave has a frequency of $50 \mathrm{~Hz}$, and $\eta=\phi=0$. The absolute values are shown with semi-log scales. (a) Absolute value of the reflection and transmission coefficients ( $P$ and $S$ waves); (b) phase angle of the reflection and transmission coefficients ( $P$ and $S$ waves). 
$z=h$. Our results are consistent with those of [Carcione, 2007, Eq. (6.215)].

\section{Case 3. Media with the same frame and three different fluids}

Here we validate our expressions against the reflection and transmission coefficients for a single planar interface separating two half spaces of saturated porous media. To perform the comparison with the results published in Santos et al. (2004), we set $h=0 \mathrm{~cm}$ in Eq. (28) to recover Eq. (19) of that paper, i.e., the thin layer model in Fig. 1 reduces to the case of two Biot media in contact. The frames in the three poroelastic media $\Omega_{1}, \Omega_{2}$, and $\Omega_{3}$ in Fig. 1 are defined by $K_{m}=8.66 \mathrm{GPa}, K_{s}=37 \mathrm{GPa}, \mu=6.5 \mathrm{GPa}, \phi=0.297$, $\kappa=1.9 \mathrm{D}$, and $\rho_{s}=2.65 \mathrm{~g} / \mathrm{cm}^{3}$. The saturant fluids are water in $\Omega_{1}$, gas in $\Omega_{2}$ and oil in $\Omega_{3}$, with properties given in Table I.
The incident plane wave is type-II of frequency $50 \mathrm{kHz}$. Figure 4 shows the magnitude of the reflection coefficients as a function of the incidence angle for the different types of waves and three different values of the layer thickness, $h=0.1 \mathrm{~cm}$ ( $2 \%$ of the wavelength of the type-I wave in the layer), $h=0.4 \mathrm{~cm}$ and $h=0 \mathrm{~cm}$. For the type-I and II waves, the magnitude of the reflection coefficients decreases as $h$ increases, while the opposite behavior is observed for shear waves. Besides, for type-I and II waves, there is a critical angle between 10 and $20 \mathrm{deg}$; for shear waves two critical angles exists for the case $h=0$. The transmission coefficients of the three types of waves behave similarly to the reflection coefficients. The plots are not included for brevity. Note that in Fig. 4, for the case $h=0$, we get a perfect agreement with the results reported in Figs. 6, 7, and 8 of Santos et al. (2004).
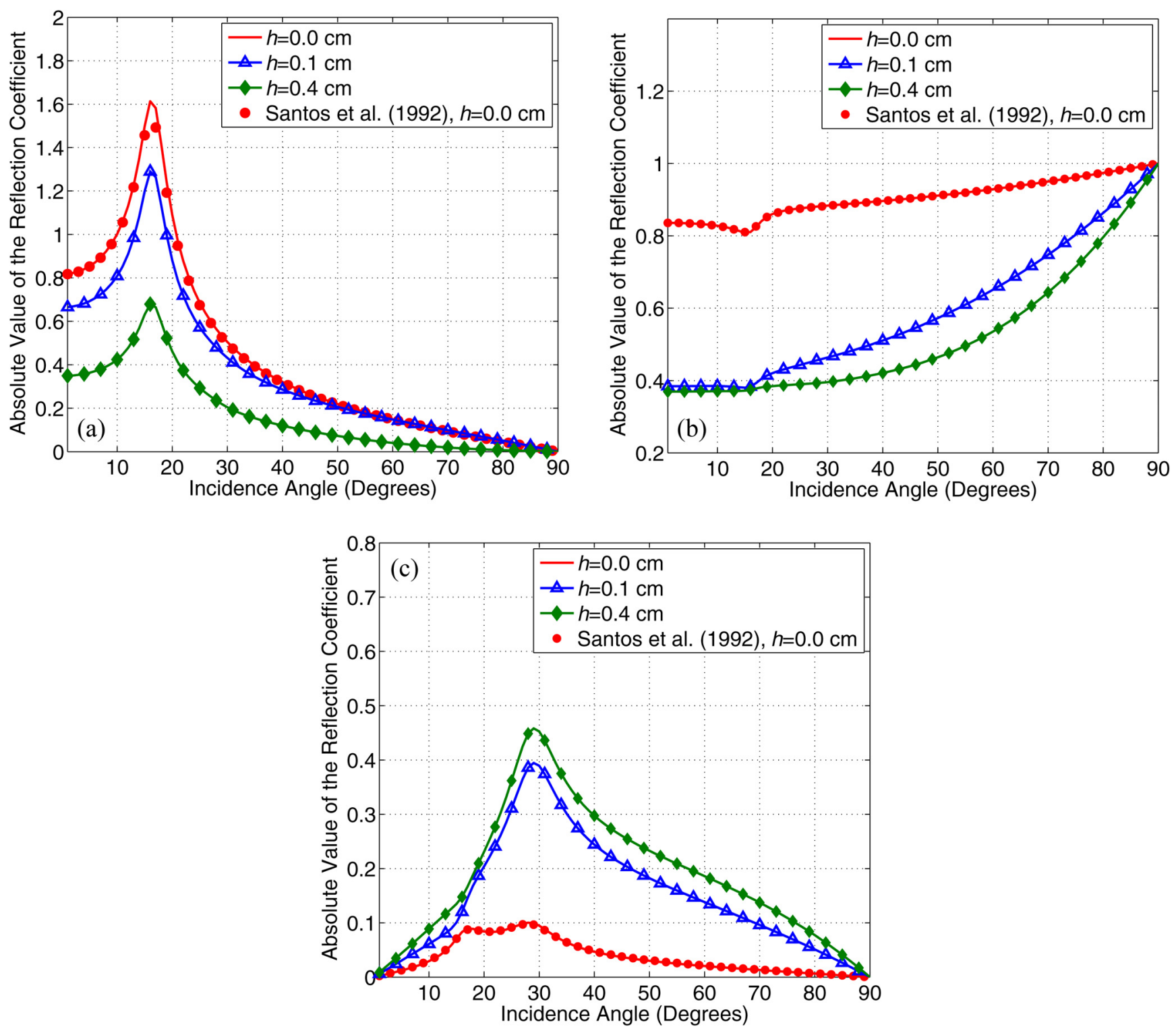

FIG. 4. (Color online) Absolute value of the reflection coefficients of the type I, type II and shear waves as a function of the incidence angle corresponding to three different layer thicknesses $h$. The incident wave is a type II $P$-wave of frequency $50 \mathrm{kHz}$. (a) Absolute value (type I wave); (b) absolute value (type II wave); (c) absolute value (shear wave). 


\section{Case 4. Three media saturated with the same fluid}

The regions $\Omega_{1}$ and $\Omega_{3}$ (the background) are described by the properties $K_{m}=1.17 \mathrm{GPa}, \quad K_{s}=37 \mathrm{GPa}$, $\mu=1.4 \mathrm{GPa}, \phi=0.25$, and $\kappa=0.18 \mathrm{D}$. On the other hand, the medium $\Omega_{2}$ is a highly permeable and compliant porous layer of porosity $\phi=0.5$ with $K_{m}=0.58 \mathrm{GPa}$ and $\mu=0.6 \mathrm{GPa}$ determined from the Krief model (Krief et al., 1990):

$$
\frac{K_{m}}{K_{s}}=\frac{\mu}{\mu_{s}}=(1-\phi)^{3 /(1-\phi)},
$$

with $K_{s}=37 \mathrm{GPa}$ and $\mu_{s}=44 \mathrm{GPa}$. The permeability in $\Omega_{2}$ is computed from the relation (Carcione et al., 2000):

$$
\kappa=\frac{r_{g}^{2} \phi^{3}}{45(1-\phi)^{2}},
$$
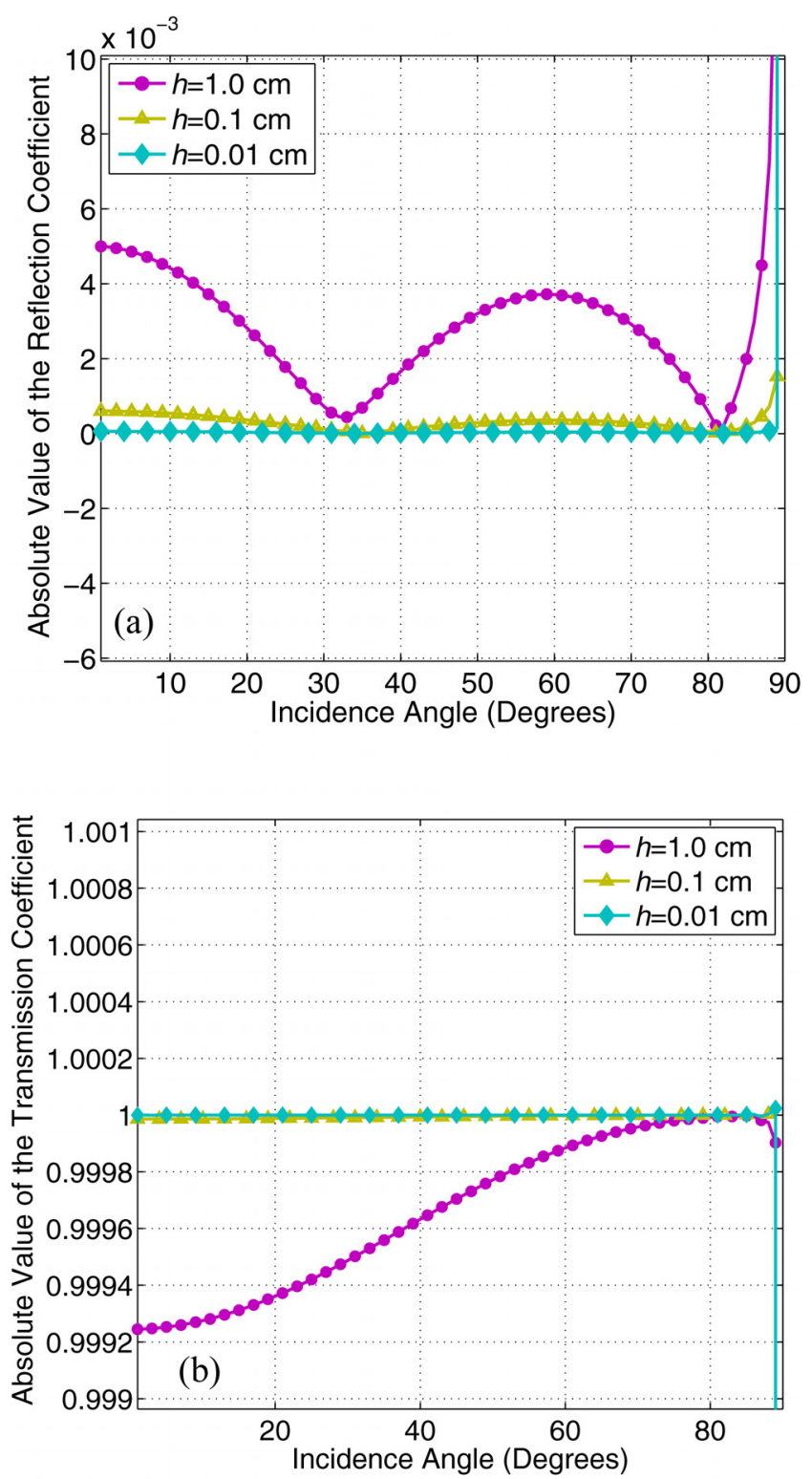

where $r_{g}=20 \mu \mathrm{m}$ is the average radius of the grains, giving a value of $2.22 \mathrm{D}$. The layer thickness $h$ varies from $0.05 \%$ to $0.0005 \%$ of the type-I $P$-wave wavelength of the background. The three media are saturated with water.

Figure 5 shows the magnitude and phase of the reflection and transmission coefficients as a function of the incidence angle. The incident plane wave is a type-I $P$ wave of frequency $50 \mathrm{~Hz}$. The reflection coefficients decrease as $h$ tends to zero, i.e., when the three-layer system approaches a single medium with identical properties, and tends to one as the angle tends to $90 \mathrm{deg}$; both coefficients exhibit a critical angle at about $26 \mathrm{deg}$, and polarity changes can be observed in the phases. The transmission coefficient remains approximately constant, except at $90 \mathrm{deg}$, where it approaches zero.

Figure 6 shows the magnitude and phase of the reflection and transmission coefficients of the type-II $P$ waves as a function of the incidence angle. The magnitudes decrease as
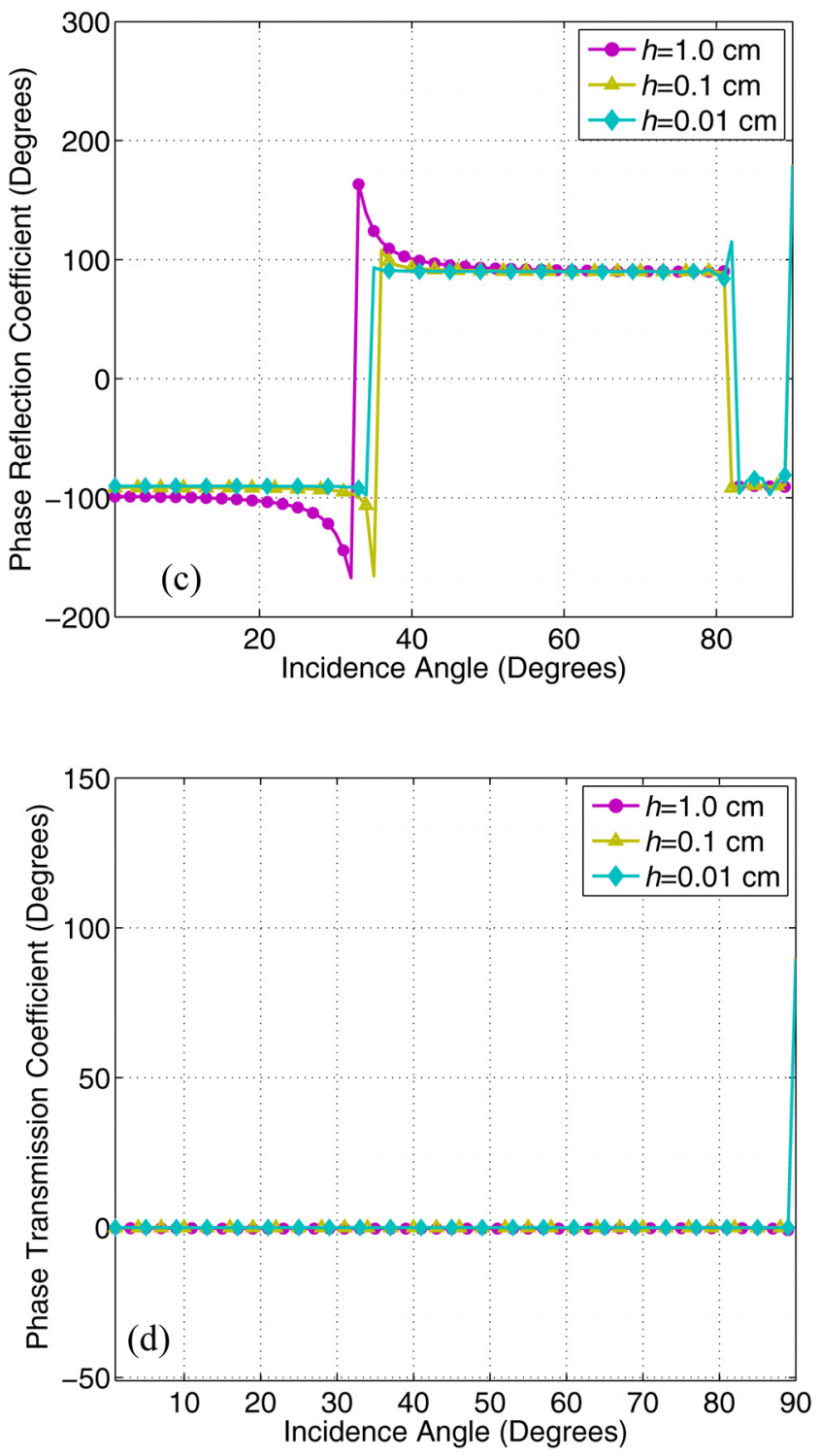

FIG. 5. (Color online) Reflection and transmission coefficients of type I waves as a function of the incidence angle for three different thicknesses $h$. The incident wave is a type I $P$-wave of frequency $50 \mathrm{~Hz}$. (a) Absolute value of the reflection coefficient; (b) absolute value of the transmission coefficient; (c) phase of the reflection coefficient; (d) phase of the transmission coefficient. 

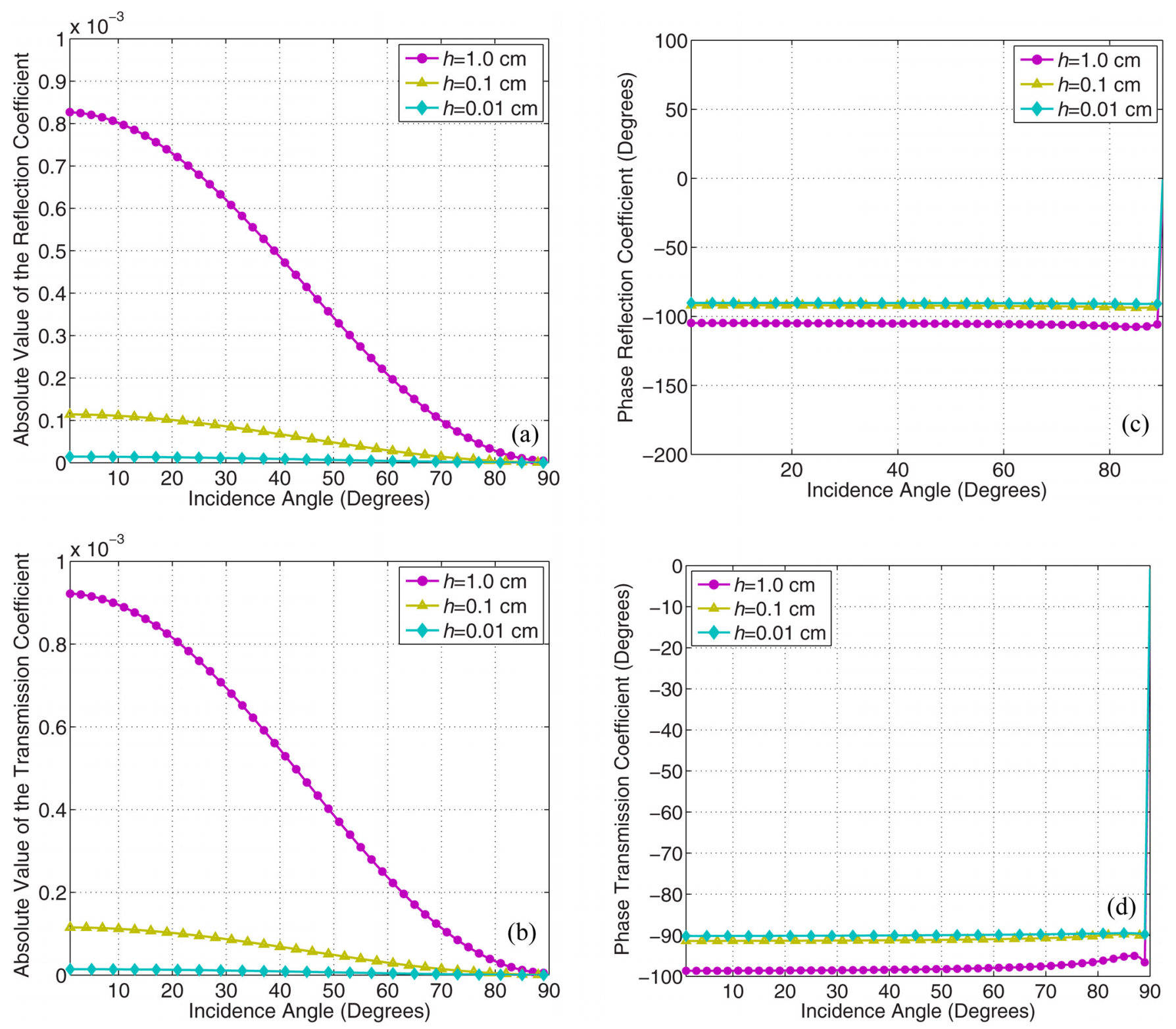

FIG. 6. (Color online) Reflection and transmission coefficients of type II waves as a function of the incidence angle for three different thicknesses $h$. The incident wave is a type I $P$-wave of frequency $50 \mathrm{~Hz}$. (a) Absolute value of the reflection coefficient; (b) absolute value of the transmission coefficient; (c) phase of the reflection coefficient; (d) phase of the transmission coefficient.

the angle of incidence tends to zero, while the phases remain approximately constant. Figure 7 displays the magnitude and phase of the reflection and transmission coefficients of the shear waves as a function of the incidence angle. The reflection and transmission coefficients have no critical angles, and the phases remains constant except for angles near $90 \mathrm{deg}$.

\section{CONCLUSIONS}

We have solved the scattering problem for waves incident in a fluid-saturated porous system composed of a plane layer embedded between two half-spaces, where the media are described by Biot's theory of poroelasticity. The results are validated with known limit cases, such as zero porosity (elastic solids) and $100 \%$ porosity (inviscid fluids), and the case of a single interface between two poroelastic half-spaces when the thickness of the layer tends to zero.
The equations predict all the wave conversions, critical angles and polarity changes, depending on the type of incident wave. Specific cases have been studied here, such as is a highly permeable and compliant porous layer. The equations can be used to study many practical cases, as for instance, the seismic response of fractures in sandstone ad carbonate reservoirs and the AVO effects of a reservoir layer as a function of the type of fluid and characteristics of the porous skeleton, such as the porosity and the permeability.

\section{ACKNOWLEDGMENTS}

This work was partially supported by the Agencia Nacional de Promoción Científica y Tecnológica, Argentina through FONARSEC FSTIC 06/10 and by CONICET from PIP 0952. J.M.C. was partially funded by the CO2Monitor project. 

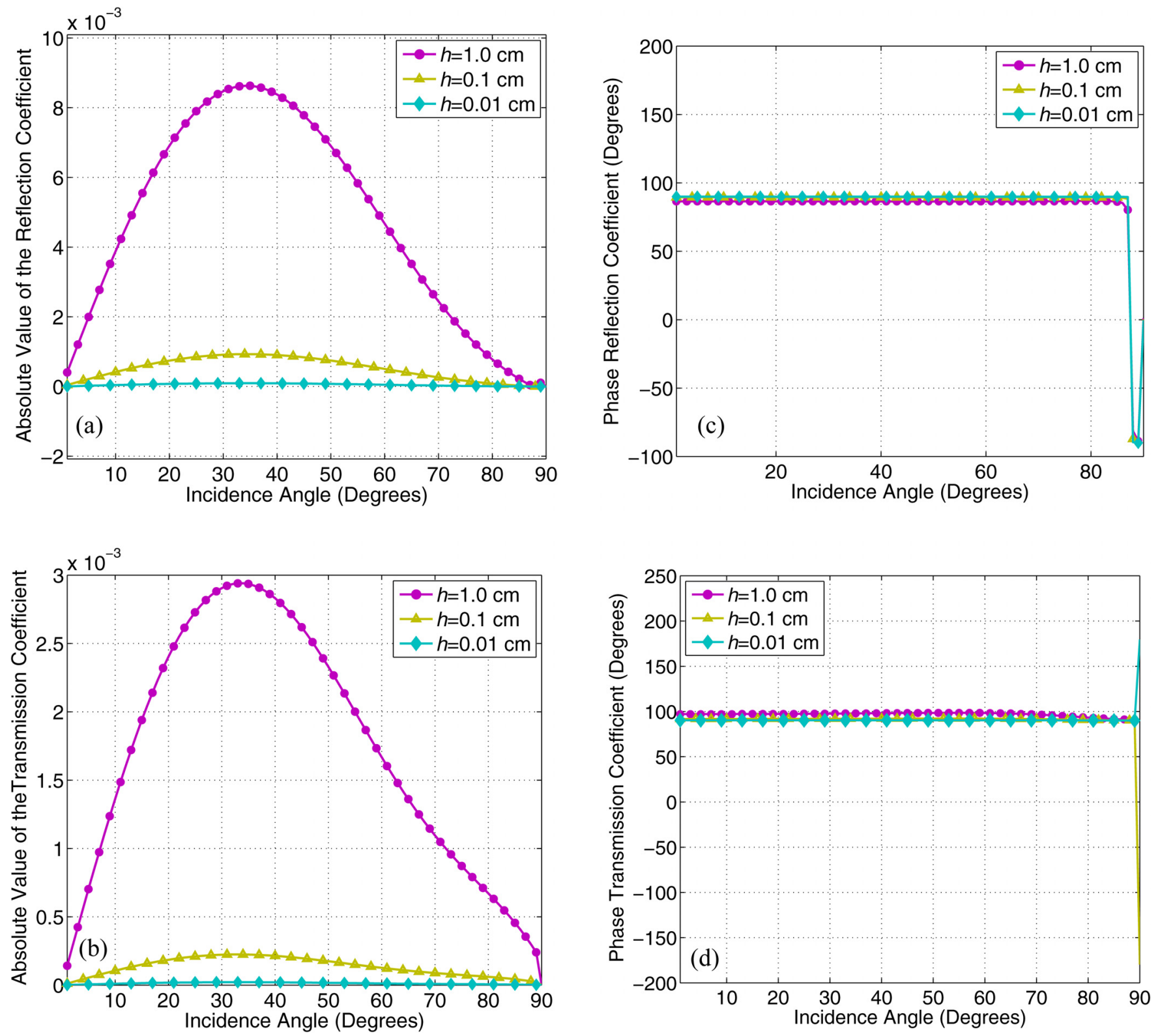

FIG. 7. (Color online) Reflection and transmission coefficients of shear waves as a function of the incidence angle for three different thickness $h$. The incident wave is a type I $P$-wave of frequency $50 \mathrm{~Hz}$. (a) Absolute value of the reflection coefficient; (b) absolute value of the transmission coefficient; (c) phase of the reflection coefficient; (d) phase of the transmission coefficient.

\section{APPENDIX A: LINEAR SYSTEMS}

Here, we report the linear equations for the unknown amplitude of the reflected and transmitted waves. First, application of the boundary conditions (17)-(22) at $z=0$ yields the linear system

$$
\begin{aligned}
& -\chi A_{i 1}^{(1)}-\chi A_{r 1}^{(1)}-\chi A_{r 2}^{(1)}+\beta_{r s}^{(1)} A_{r s}^{(1)} \\
& =-\chi A_{t 1}^{(2)}-\chi A_{t 2}^{(2)}+\beta_{t s}^{(2)} A_{t s}^{(2)}-\chi A_{r 1}^{(2)}-\chi A_{r 2}^{(2)}+\beta_{r s}^{(2)} A_{r s}^{(2)}, \\
& -\beta_{i 1}^{(1)} A_{i 1}^{(1)}-\beta_{r 1}^{(1)} A_{r 1}^{(1)}-\beta_{r 2}^{(1)} A_{r 2}^{(1)}-\chi A_{r s}^{(1)} \\
& =-\beta_{t 1}^{(2)} A_{t 1}^{(2)}-\beta_{t 2}^{(2)} A_{t 2}^{(2)}-\chi A_{t s}^{(2)} \\
& \quad-\beta_{r 1}^{(2)} A_{r 1}^{(2)}-\beta_{r 2}^{(2)} A_{r 2}^{(2)}-\chi A_{r s}^{(2)},
\end{aligned}
$$

$$
\begin{aligned}
& A_{i 1}^{(1)} \zeta_{i 1}^{(1)}+A_{r 1}^{(1)} \zeta_{r 1}^{(1)}+A_{r 2}^{(1)} \zeta_{r 2}^{(1)}-A_{r s}^{(1)} \zeta_{r s}^{(1)} \\
&= A_{t 1}^{(2)} \zeta_{t 1}^{(2)}+A_{t 2}^{(2)} \zeta_{t 2}^{(2)}-A_{t s}^{(2)} \zeta_{t s}^{(2)} \\
&+A_{r 1}^{(2)} \zeta_{r 1}^{(2)}+A_{r 2}^{(2)} \zeta_{r 2}^{(2)}-A_{r s}^{(2)} \zeta_{r s}^{(2)} \\
&-2 \mu^{(1)} A_{i 1} \chi \beta_{i 1}^{(1)}-2 \mu^{(1)} A_{r 1} \chi \beta_{r 1}^{(1)}-2 \mu^{(1)} A_{r 2} \chi \beta_{r 2}^{(1)} \\
&-\mu^{(1)} A_{r s}\left[\chi^{2}-\left(\beta_{r s}^{(1)}\right)^{2}\right] \\
&=-2 \mu^{(2)} A_{t 1} \chi \beta_{t 1}^{(2)}-2 \mu^{(2)} A_{t 2} \chi \beta_{t 2}^{(2)} \\
& \quad-\mu^{(2)} A_{t s}\left[\chi^{2}-\left(\beta_{t s}^{(2)}\right)^{2}\right]-2 \mu^{(2)} A_{r 1} \chi \beta_{r 1}^{(2)} \\
& \quad-2 \mu^{(2)} A_{r 2} \chi \beta_{r 2}^{(2)}-\mu^{(2)} A_{r s}\left[\chi^{2}-\left(\beta_{r s}^{(2)}\right)^{2}\right],
\end{aligned}
$$




$$
\begin{aligned}
& A_{i 1}^{(1)} \xi_{i 1}^{(1)}+A_{r 1}^{(1)} \xi_{r 1}^{(1)}+A_{r 2}^{(1)} \xi_{r 2}^{(1)}=A_{t 1}^{(2)} \xi_{t 1}^{(2)}+A_{t 2}^{(2)} \xi_{t 2}^{(2)}+A_{r 1}^{(2)} \xi_{r 1}^{(2)}+A_{r 2}^{(2)} \xi_{r 2}^{(2)}, \\
& -\beta_{i 1}^{(1)} \gamma_{2}^{(1)} A_{i 1}^{(1)}-\beta_{r 1}^{(1)} \gamma_{1}^{(1)} A_{r 1}^{(1)}-\beta_{r 2}^{(1)} \gamma_{2}^{(1)} A_{r 2}^{(1)}-\chi \gamma_{r s}^{(1)} A_{r s}^{(1)} \\
& \quad=-\beta_{t 1}^{(2)} \gamma_{1}^{(2)} A_{t 1}^{(2)}-\beta_{t 2}^{(2)} \gamma_{2}^{(2)} A_{t 2}^{(2)}-\chi \gamma_{t s}^{(2)} A_{t s}^{(2)}-\beta_{r 1}^{(2)} \gamma_{1}^{(2)} A_{r 1}^{(2)}-\beta_{r 2}^{(2)} \gamma_{2}^{(2)} A_{r 2}^{(2)}-\chi \gamma_{r s}^{(2)} A_{r s}^{(2)} .
\end{aligned}
$$

Similarly, at $z=h$ we obtain

$$
\begin{aligned}
& -\chi A_{t 1}^{(3)} e^{-\mathrm{i} \beta_{t 1}^{(3)} h}-\chi A_{t 2}^{(3)} e^{-\mathrm{i} \beta_{t 2}^{(3)} h}+\beta_{t s}^{(3)} A_{t s}^{(3)} e^{-\mathrm{i} \beta_{t s}^{(3)} h}=-\chi A_{t 1}^{(2)} e^{-\mathrm{i} \beta_{t 1}^{(2)} h}-\chi A_{t 2}^{(2)} e^{-\mathrm{i} \beta_{t 2}^{(2)} h}+\beta_{t s}^{(2)} A_{t s}^{(2)} e^{-\mathrm{i} \beta_{t s}^{(2)} h}-\chi A_{r 1}^{(2)} e^{-\mathrm{i} \beta_{r 1}^{(2)} h} \\
& -\chi A_{r 2}^{(2)} e^{-\mathrm{i} \beta_{r 2}^{(2)} h}+\beta_{r s}^{(2)} A_{r s}^{(2)} e^{-\mathrm{i} \beta_{r s}^{(2)}} \\
& -\beta_{t 1}^{(3)} A_{t 1}^{(3)} e^{-\mathrm{i} \beta_{t 1}^{(3)} h}-\beta_{t 2}^{(3)} A_{t 2}^{(3)} e^{-\mathrm{i} \beta_{t 2}^{(3)} h}-\chi A_{t s}^{(3)} e^{-\mathrm{i} \beta_{t s}^{(3)} h}=-\beta_{t 1}^{(2)} A_{t 1}^{(2)} e^{-\mathrm{i} \beta_{t 1}^{(2)} h}-\beta_{t 2}^{(2)} A_{t 2}^{(2)} e^{-\mathrm{i} \beta_{t 2}^{(2)} h}-\chi A_{t s}^{(2)} e^{-\mathrm{i} \beta_{t s}^{(2)} h} \\
& -\beta_{r 1}^{(2)} A_{r 1}^{(2)} e^{-\mathrm{i} \beta_{r 1}^{(2)} h}-\beta_{r 2}^{(2)} A_{r 2}^{(2)} e^{-\mathrm{i} \beta_{r 2}^{(2)} h}-\chi A_{r s}^{(2)} e^{-\mathrm{i} \beta_{r s}^{(2)} h}, \\
& A_{t 1}^{(3)} \zeta_{t 1}^{(3)} e^{-\mathrm{i} \beta_{t 1}^{(3)} h}+A_{t 2}^{(3)} \zeta_{t 2}^{(3)} e^{-\mathrm{i} \beta_{t 2}^{(3)} h}-A_{t s}^{(3)} \zeta_{t s}^{(3)} e^{-\mathrm{i} \beta_{t s}^{(3)} h}=A_{t 1}^{(2)} \zeta_{t 1}^{(2)} e^{-\mathrm{i} \beta_{t 1} h}+A_{t 2}^{(2)} \zeta_{t 2}^{(2)} e^{-\mathrm{i} \beta_{t 2}^{(2)} h}-A_{t s}^{(2)} \zeta_{t s}^{(2)} e^{-\mathrm{i} \beta_{t s} y}+A_{r 1}^{(2)} \zeta_{r 1}^{(2)} e^{-\mathrm{i} \beta_{r 1} h} \\
& +A_{r 2}^{(2)} \zeta_{r 2}^{(2)} e^{-\mathrm{i} \beta_{r 2} h}-A_{r s}^{(2)} \zeta_{r s}^{(2)} e^{-\mathrm{i} \beta_{r s} h} \\
& -2 \mu^{(3)} A_{t 1} \chi \beta_{t 1}^{(3)} e^{-\mathrm{i} \beta_{t 1}^{(3)} h}-2 \mu^{(3)} A_{t 2} \chi \beta_{t 2}^{(3)} e^{-\mathrm{i} \beta_{t 2}^{(3)} h}-\mu^{(3)} A_{t s}\left[\chi^{2}-\left(\beta_{t s}^{(3)}\right)^{2}\right] e^{-\mathrm{i} \beta_{t s}^{(3)} h} \\
& =-2 \mu^{(2)} A_{t 1}^{(2)} \chi \beta_{t 1}^{(2)} e^{-\mathrm{i} \beta_{t 1}^{(2)} h}-2 \mu^{(2)} A_{t 2}^{(2)} \chi \beta_{t 2}^{(2)} e^{-\mathrm{i} \beta_{t 2}^{(2)} h}-\mu^{(2)} A_{t s}^{(2)}\left[\chi^{2}-\left(\beta_{t s}^{(2)}\right)^{2}\right] e^{-\mathrm{i} \beta_{t s}^{(2)} h} \\
& -2 \mu^{(2)} A_{r 1}^{(2)} \chi \beta_{r 1}^{(2)} e^{-\mathrm{i} \beta_{r 1}^{(2)} h}-2 \mu^{(2)} A_{r 2}^{(2)} \chi \beta_{r 2}^{(2)} e^{-\mathrm{i} \beta_{r 2}^{(2)} h}-\mu^{(2)} A_{r s}^{(2)}\left[\chi^{2}-\left(\beta_{r s}^{(2)}\right)^{2}\right] e^{-\mathrm{i} \beta_{r s}^{(2)} h}, \\
& A_{t 1}^{(3)} \xi_{t 1}^{(3)} e^{-\mathrm{i} \beta_{t 1}^{(3)} h}+A_{t 2}^{(3)} \xi_{t 2}^{(3)} e^{-\mathrm{i} \beta_{t 2}^{(3)} h}=A_{t 1}^{(2)} \xi_{t 1}^{(2)} e^{-\mathrm{i} \beta_{t 1}^{(2)} h}+A_{t 2}^{(2)} \xi_{t 2}^{(2)} e^{-\mathrm{i} \beta_{t 2}^{(2)} h}+A_{r 1}^{(2)} \xi_{r 1}^{(2)} e^{-\mathrm{i} \beta_{r 1}^{(2)} h}+A_{r 2}^{(2)} \xi_{r 2}^{(2)} e^{-\mathrm{i} \beta_{r 2}^{(2)} h} \text {, } \\
& -\beta_{t 1}^{(3)} \gamma_{1}^{(3)} A_{t 1}^{(3)} e^{-\mathrm{i} \beta_{t 1}^{(3)} h}-\beta_{t 2}^{(3)} \gamma_{2}^{(3)} A_{t 2}^{(3)} e^{-\mathrm{i} \beta_{t 2}^{(3)} h}-\chi \gamma_{t s}^{(3)} A_{t s}^{(3)} e^{-\mathrm{i} \beta_{t s}^{(3)} h} \\
& =-\beta_{t 1}^{(2)} \gamma_{1}^{(2)} A_{t 1}^{(2)} e^{-\mathrm{i} \beta_{t 1}^{(2)} h}-\beta_{t 2}^{(2)} \gamma_{2}^{(2)} A_{t 2}^{(2)} e^{-\mathrm{i} \beta_{t 2}^{(2)} h}-\chi \gamma_{t s}^{(2)} A_{t s}^{(2)} e^{-\mathrm{i} \beta_{t s}^{(2)} h} \\
& -\beta_{r 1}^{(2)} \gamma_{1}^{(2)} A_{r 1}^{(2)} e^{-\mathrm{i} \beta_{r 1}^{(2)} h}-\beta_{r 2}^{(2)} \gamma_{2}^{(2)} A_{r 2}^{(2)} e^{-\mathrm{i} \beta_{r 2}^{(2)} h}-\chi \gamma_{r s}^{(2)} A_{r s}^{(2)} e^{-\mathrm{i} \beta_{r s}^{(2)} h} \text {. }
\end{aligned}
$$

The coefficients of the systems (A1)-(A6) and (A7)-(A12) are given by

$$
\begin{aligned}
& \beta_{r j}^{(n)}=-\sqrt{\left(q_{r j}^{(n)}\right)^{2}-\chi^{2}}, \quad j=1,2, s, \quad n=1,2, \\
& \beta_{t j}^{(n)}=-\sqrt{\left(q_{t j}^{(n)}\right)^{2}-\chi^{2}}, \quad j=1,2, s, \quad n=2,3, \\
& \zeta_{l j}^{(n)}=-\left(q_{l j}^{(n)}\right)^{2}\left(H_{c}^{(n)}+D^{(n)} \gamma_{l j}^{(n)}\right)+2 \mu^{(n)} \chi^{2}, \quad j=1,2, \quad n=1,2,3, \quad l=i, r, t, \\
& \zeta_{l s}^{(n)}=2 \mu^{(n)} \chi \beta_{l s}^{(n)}, \quad n=1,2,3, \quad l=r, t, \\
& \xi_{l j}^{(n)}=\left(D^{(n)}+M^{(n)} \gamma_{l j}^{(n)}\right)\left(q_{l j}^{(n)}\right)^{2}, \quad j=1,2, \quad n=1,2,3, \quad l=i, r, t .
\end{aligned}
$$

Now, using Eqs. (3) and (5), we obtain the wave numbers $q_{l j}^{(n)}, n=1,2,3, l=i, r, t$, and $j=1,2, s$ :

$$
\begin{aligned}
& q_{r 1}^{(n)}=\sqrt{\frac{-F^{(n)}-\sqrt{\left(F^{(n)}\right)^{2}-4 G^{(n)} K^{(n)}}}{2 G^{(n)}}}, \quad n=1,2,3, \\
& q_{r 2}^{(n)}=\sqrt{\frac{-F^{(n)}+\sqrt{\left(F^{(n)}\right)^{2}-4 G^{(n)} K^{(n)}}}{2 G^{(n)}}}, \quad n=1,2,3, \\
& q_{r s}^{(1)}=\sqrt{N^{(n)}-i V^{(n)}}, \quad n=1,2,3, \\
& q_{r 1}^{(1)}=q_{i 1}^{(1)}, \quad \\
& q_{r j}^{(n)}=q_{t j}^{(n)}, \quad j=1,2, s, \quad n=1,2,3 .
\end{aligned}
$$

Here

$$
G^{(n)}=\left(M^{(n)} H_{c}^{(n)}\right)-\left(D^{(n)}\right)^{2}, \quad n=1,2,3,
$$




$$
\begin{aligned}
& F^{(n)}=\omega^{2}\left[2 \rho_{f}^{(n)} D^{(n)}-H_{c}^{(n)} g^{(n)}-\rho_{b}^{(n)} M^{(n)}\right]+\mathrm{i} H_{c}^{(n)} b^{(n)} \omega, \quad n=1,2,3, \\
& K^{(n)}=\omega^{4}\left[\rho_{b}^{(n)} g^{(n)}-\left(\rho_{f}^{(n)}\right)^{2}\right]-\mathrm{i} \rho_{b}^{(n)} b^{(n)} \omega^{3}, \quad n=1,2,3, \\
& N^{(n)}=\frac{\omega^{2}}{\mu^{(n)}}\left[\rho_{b}^{(n)}-\frac{\left(\rho_{f}^{(n)}\right)^{2} \omega^{2} g^{(n)}}{\left(g^{(n)}\right)^{2} \omega^{2}+\left(b^{(n)}\right)^{2}}\right], \quad n=1,2,3, \\
& V^{(n)}=\left(\rho_{f}^{(n)}\right)^{2} \frac{\omega^{3} b^{(n)}}{\mu^{(n)}\left(\left(g^{(n)}\right)^{2} \omega^{2}+\left(b^{(n)}\right)^{2}\right)}, \quad n=1,2,3 .
\end{aligned}
$$

\section{APPENDIX B: FINAL SYSTEM OF EQUATIONS}

Each of the matrices in system (28) are defined by

$$
\mathbf{A}_{1}=\left(\begin{array}{cccccc}
-\chi & -\chi & \beta_{r s}^{(1)} & 0 & 0 & 0 \\
-\beta_{r 1}^{(1)} & -\beta_{r 2}^{(1)} & -\chi & 0 & 0 & 0 \\
\zeta_{r 1}^{(1)} & \zeta_{r 2}^{(1)} & -\zeta_{r s}^{(1)} & 0 & 0 & 0 \\
-2 \mu^{(1)} \chi \beta_{r 1}^{(1)} & -2 \mu^{(1)} \chi \beta_{r 2}^{(1)} & -\mu^{(1)}\left[\chi^{2}-\left(\beta_{r s}^{(1)}\right)^{2}\right] & 0 & 0 & 0 \\
\xi_{r 1}^{(1)} & \xi_{r 2}^{(1)} & 0 & 0 & 0 & 0 \\
-\gamma_{r 1}^{(1)} \beta_{r 1}^{(1)} & -\gamma_{r 2}^{(1)} \beta_{r 2}^{(1)} & -\gamma_{r s}^{(1)} \chi & 0 & 0 & 0
\end{array}\right)
$$

and

$$
\mathbf{A}_{3}=\left(\begin{array}{cccccc}
0 & 0 & 0 & -\chi e^{-\mathrm{i} \beta_{t 1}^{(3)} h} & -\chi e^{-\mathrm{i} \beta_{t 2}^{(3)} h} & \beta_{t s}^{(3)} e^{-\mathrm{i} \beta_{t s}^{(3)} h} \\
0 & 0 & 0 & -\beta_{t 1}^{(3)} e^{-\mathrm{i} \beta_{t 1}^{(3)} h} & -\beta_{t 2}^{(3)} e^{-\mathrm{i} \beta_{t 2}^{(3)} h} & -\chi e^{-\mathrm{i} \beta_{t s}^{(3)} h} \\
0 & 0 & 0 & \zeta_{t 1}^{(3)} e^{-\mathrm{i} \beta_{t 1}^{(3)} h} & \zeta_{t 2}^{(3)} e^{-\mathrm{i} \beta_{t 2}^{(3)} h} & -\zeta_{t s}^{(3)} e^{-\mathrm{i} \beta_{t s}^{(3)} h} \\
0 & 0 & 0 & -2 \mu^{(3)} \chi \beta_{t 1}^{(3)} e^{-\mathrm{i} \beta_{t 1}^{(3)} h} & -2 \mu^{(3)} \chi \beta_{t 2}^{(3)} e^{-\mathrm{i} \beta_{t 2}^{(3)} h} & -\mu^{(3)}\left[\chi^{2}-\left(\beta_{t s}^{(3)}\right)^{2}\right] e^{-\mathrm{i} \beta_{t s}^{(3)} h} \\
0 & 0 & 0 & \xi_{t 1}^{(3)} e^{-\mathrm{i} \beta_{t 1}^{(3)} h} & \xi_{t 2}^{(3)} e^{-\mathrm{i} \beta_{t 2}^{(3)} h} & 0 \\
0 & 0 & 0 & -\gamma_{t 1}^{(3)} \beta_{t 1}^{(3)} e^{-\mathrm{i} \beta_{t 1}^{(3)} h} & -\gamma_{t 2}^{(3)} \beta_{t 2}^{(3)} e^{-\mathrm{i} \beta_{t 2}^{(3)} h} & -\gamma_{t s}^{(3)} \chi e^{-\mathrm{i} \beta_{t s}^{(3)} h}
\end{array}\right) .
$$

Finally, $\mathbf{B}=\mathbf{T}(0) *[\mathbf{T}(h)]^{-1}$ and $T(z)=\mathbf{S}_{1} * \mathbf{S}_{2}(z)$ being

$$
\mathbf{S}_{1}=\left(\begin{array}{cccccc}
-\chi & -\chi & \beta_{t s}^{(2)} & -\chi & -\chi & \beta_{r s}^{(2)} \\
-\beta_{t 1}^{(2)} & -\beta_{t 2}^{(2)} & -\chi & -\beta_{r 1}^{(2)} & -\beta_{r 2}^{(2)} & -\chi \\
\zeta_{t 1}^{(2)} & \zeta_{t 2}^{(2)} & -\zeta_{t s}^{(2)} & \zeta_{r 1}^{(2)} & \zeta_{r 2}^{(2)} & -\zeta_{r s}^{(2)} \\
-2 \mu^{(2)} \chi \beta_{t 1}^{(2)} & -2 \mu^{(2)} \chi \beta_{t 2}^{(2)} & -\mu^{(2)}\left[\chi^{(2)}-\left(\beta_{t s}^{(2)}\right)^{2}\right] & -2 \mu^{(2)} \chi \beta_{r 1}^{(2)} & -2 \mu^{(2)} \chi \beta_{r 2}^{(2)} & -\mu^{(2)}\left[\chi^{(2)}-\left(\beta_{r s}^{(2)}\right)^{2}\right] \\
\xi_{t 1}^{(2)} & \xi_{t 2}^{(2)} & 0 & \xi_{r 1}^{(2)} & \xi_{r 2}^{(2)} & 0 \\
-\gamma_{t 1}^{(2)} l_{t 1}^{(2)} & -\gamma_{t 2}^{(2)} l_{t 2}^{(2)} & -\gamma_{t s}^{(2)} \chi & -\gamma_{r 1}^{(2)} l_{r 1}^{(2)} & -\gamma_{r 2}^{(2)} l_{r 2}^{(2)} & -\gamma_{r s}^{(2)} \chi
\end{array}\right)
$$

and

$$
\mathbf{S}_{2}(z)=\left(\begin{array}{cccccc}
e^{-\mathrm{i} \beta_{t 1}^{(2)} z} & 0 & 0 & 0 & 0 & 0 \\
0 & e^{-\mathrm{i} \beta_{t 2}^{(2)} z} & 0 & 0 & 0 & 0 \\
0 & 0 & e^{-\mathrm{i} \beta_{t s}^{(2)} z} & 0 & 0 & 0 \\
0 & 0 & 0 & e^{-\mathrm{i} \beta_{r 1}^{(2)} z} & 0 & 0 \\
0 & 0 & 0 & 0 & e^{-\mathrm{i} \beta_{r 2}^{(2)} z} & 0 \\
0 & 0 & 0 & 0 & 0 & e^{-\mathrm{i} \beta_{r s}^{(2)} z}
\end{array}\right) .
$$


Allard, J. F., Bourdier, R., and Depollier, C. (1986). "Biot waves in layered media," J. Appl. Phys. 60, 1926-1929.

Bakke, N. E., and Ursin, B. (1998). "Thin-bed AVO effects," Geophys. Prospect. 46, 571-587.

Berryman, J. G. (1980). "Confirmation of Biot's theory," Appl. Phys. Lett. 37, 382-384.

Berryman, J. G. (1982). "Elastic waves in fluid-saturated porous media," in Macroscopic Properties of Disordered Media (Springer Berlin, Heidelberg), pp. 38-50.

Biot, M. A. (1956). "Theory of propagation of elastic waves in a fluid-saturated porous solid. I. Low-frequency range," J. Acoust. Soc. Am. 28, 168-178.

Biot, M. A. (1962). "Mechanics of deformation and acoustic propagation in porous media," J. Appl. Phys. 33, 1482-1498.

Brekhovskikh, L. M. (1980). Waves in Layered Media, 2nd ed. (Academic, New York), 503 pp.

Carcione, J. M. (1996). "Elastodynamics of a non-ideal interface: Application to crack and fracture scattering," J. Geophys. Res.: Solid Earth 101, 28177-28188.

Carcione, J. M. (1997). "Reflection and transmission of $q P-q S$ plane waves at a plane boundary between viscoelastic transversely isotropic media," Geophys. J. Int. 129, 669-680.

Carcione, J. M. (1998). "Scattering of elastic waves by a plane crack of finite width in a transversely isotropic medium," Int. J. Numer. Anal. Methods Geomech. 22, 263-275.

Carcione, J. M. (2001). "Amplitude variations with offset of pressure-seal reflections," Geophysics 66, 283-293.

Carcione, J. M. (2007). "Wave fields in real media: Wave propagation in anisotropic, anelastic, porous and electromagnetic media," in Handbook of Geophysical Exploration, 2nd ed., revised and extended (Elsevier, Oxford), Vol. 38, 538 pp.

Carcione, J. M., Gurevich, B., and Cavallini, F. (2000). "A generalized Biot-Gassmann model for the acoustic properties of shaley sandstones," Geophys. Prospect. 48, 539-557.

Carcione, J. M., Morency, C., and Santos, J. E. (2010). "Computational poroelasticity-A review," Geophysics 75, A229-A243.

Carcione, J. M., and Picotti, S. (2012). "Reflection and transmission coefficients of a fracture in transversely isotropic media," Stud. Geophys. Geod. 56, 307-322.

Carcione, J. M., Santos, J. E., Ravazzoli, C. L., and Helle, H. B. (2003). "Wave simulation in partially frozen porous media with fractal freezing conditions," J. Appl. Phys. 94, 7839-7847.

Chung, H. M., and Lawton, D. C. (1995a). "Amplitude responses of thin beds: Sinusoidal approximation versus Ricker approximation," Geophysics 60, 223-230.

Chung, H. M., and Lawton, D. C. (1995b). "Frequency characteristics of seismic reflections from thin beds," Can. J. Explor. Geophys. 31, 32-37.

Dutta, N. C., and Odé, H. (1979a). "Attenuation and dispersion of compressional waves in fluid-filled porous rocks with partial gas saturation (White model)-Part I: Biot theory," Geophysics 44, 1777-1788.
Dutta, N. C., and Odé, H. (1979b). "Attenuation and dispersion of compressional waves in fluid-filled porous rocks with partial gas saturation (White model) - Part II: Results," Geophysics 44, 1789-1805.

Dutta, N. C., and Odé, H. (1983). "Seismic reflections from a gas-water contact," Geophysics 48, 14-32.

Fellah, M., Fellah, Z. E. A., Mitri, F. G., Ogam, E., and Depollier, C. (2013). "Transient ultrasound propagation in porous media using Biot theory and fractional calculus: Application to human cancellous bone," J. Acoust. Soc. Am. 133, 1867-1881.

Jocker, J., and Smeulders, D. (2009). "Ultrasonic measurement on poroelastic slabs: Determination of reflection and transmission coefficients and processing for Biot input parameters," Ultrasonics 49, 319-330.

Johnson, D. L., Plona, T. J., and Kojima, H. (1994). "Probing porous media with first and second sound II. Acoustic properties of water saturated porous media," J. Appl. Phys. 76, 115-125.

Juhlin, C., and Young, R. (1993). "Implication of thin layers for amplitude variation with offset (AVO) studies," Geophysics 58, 1200-1204.

Krief, M., Garat, J., Stellingwerff, J., and Ventre, J. (1990). “A petrophysical interpretation using the velocities of $P$ and $S$ waves (full waveform sonic)," Log Anal. 31, 355-369.

Liu, L., and Schmitt, D. R. (2003). "Amplitude and AVO responses of a single thin bed," Geophysics 68, 1161-1168.

Pilant, W. L. (1979). Elastic Waves in the Earth (Elsevier Science, Amsterdam), 493 pp.

Plona, T. (1980) "Observation of a second bulk compressional wave in a porous medium at ultrasonic frequencies," Appl. Phys. Lett. 36, 259-261.

Pride, S. R., Tromeur, E., and Berryman, J. G. (2002). "Biot slow-wave effects in stratified rock," Geophysics 67, 271-281.

Quintal, B., Schmalholz, S. M., and Podladchikov, Y. Y. (2009). "Low-frequency reflections from a thin layer with high attenuation caused by interlayer flow," Geophysics 74, N14-N22.

Rubino, J. G., Ravazzoli, C. L., and Santos, J. E. (2006). "Reflection and transmission of waves in composite porous media: A quantification of energy conversions involving slow waves," J. Acoust. Soc. Am. 120, $2425-2436$.

Santos, J. E., Corbero, J. M., Ravazzoli, C. L., and Hensley, J. L. (1992). "Reflection and transmission coefficients in fluid-saturated porous media," J. Acoust. Soc. Am. 91, 1911-1923.

Santos, J. E., Ravazzoli, C. L., and Carcione, J. M. (2004). "A model for wave propagation in a composite solid matrix saturated by a single-phase fluid," J. Acoust. Soc. Am. 115, 2749-2760.

Schmidt, H., and Tango, G. (1986). "Efficient global matrix approach to the computation of synthetic seismograms," Geophys. J. R. Astron. Soc. 84, 331-359.

Widess, M. B. (1973) "How thin is a thin bed?," Geophysics 38, $1176-1180$.

Wu, K., Xue, Q., and Adler, L. (1990). "Reflection and transmission of elastic waves from a fluid-saturated porous solid boundary," J. Acoust. Soc. Am. 87, 2346-2358. 